\title{
Force analysis and simulation of a remote inspection robot for EAST
}

\author{
Zun $\mathrm{Wu}^{1,2}$,Baoyuan $\mathrm{Wu}^{2}$, Qiang Zhang ${ }^{2}$,Zengfu Wang ${ }^{1,2, *}$ \\ ${ }^{1}$ Institute of Intelligent Machines, Chinese Academy of Sciences, Hefei, 230031, China \\ ${ }^{2}$ Department of Automation, University of Science and Technology of China, Hefei, 230026, China \\ *Corresponding Author, Email address: zfwang@ustc.edu.cn
}

Keywords: EAST, in-vessel inspection, static analysis, robot

\begin{abstract}
Experimental Advanced Superconducting Tokamak(EAST), which is a full superconducting tokamak with D-shape vacuum vessel, has been set up by ASIPP since 2006. When the harsh working condition especially the radiation is taken into consideration, it's difficult to obtain the information about the in-vessel components timely. The remote inspection robot system, named the long reach robot, is designed for the full volume inspection for the EAST vacuum vessel(VV). It is a serial-link robot with high cantilever structure, sufficient degree of freedoms(DOFs). It's essential to investigate the force performance of the device because of some components are suffered high force and toque. The force analysis and simulation presented in this paper indicate that the long reach robot has high force sufficient strength and can work properly.
\end{abstract}

\section{Introduction}

EAST is a fusion device with non-circle cross-section vacuum vessel (VV) [1]. It's essential to acquire the information of $\mathrm{VV}$ and the state of in-vessel components for guiding the operation and avoiding accidents. Considering the harsh working condition (high temperature, vacuum and radiation), it is difficult to inspect the vacuum vessel $[1,2]$.

The remote inspection robot, named the long reach robot, is presented for the full volume inspection of EAST VV. It can perform the inspection task under the environment constraints without breaking in-vessel condition. Considering the length in full extension and the limited size tube diameter, the components near the base are suffered high force and torque. Therefore, it's important to study the force performance during the inspection process. The force analysis is needed by the reason of ensuring the safety of the whole device.

This paper emphasizes on the force analysis and simulation of the long reach robot. The internal force and torque in the joints and links is given based on the energy analysis and structural analysis. Besides, the force simulation is presented via the finite element(FE) method. 


\section{Description of the long reach robot $[3,4]$}

Considering the structure of EAST VV (shown in Fig.1) and the geometric parameters[1], the remote inspection robot should have large workspace and high flexibility.

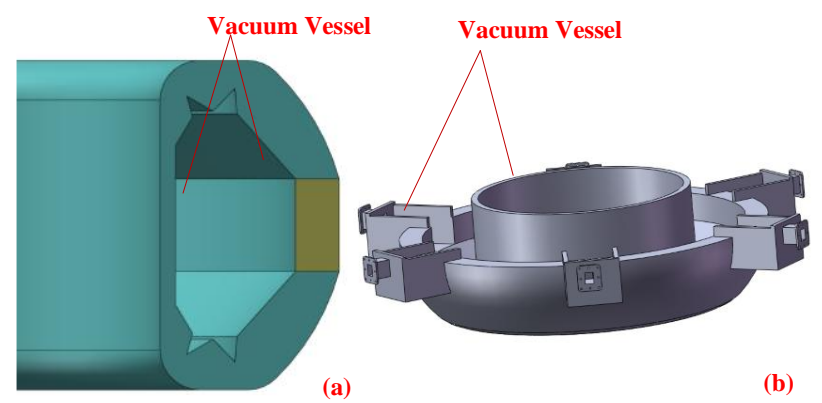

Fig. 1: The 3D model of EAST vacuum vessel: (a) Front view, (b) Top view

According to the conceptual design presented previously, the long reach robot should penetrate into the vacuum vessel through a limited size port in an equatorial plane, inspect the full volume vacuum vessel and move out the cask after inspection [3].

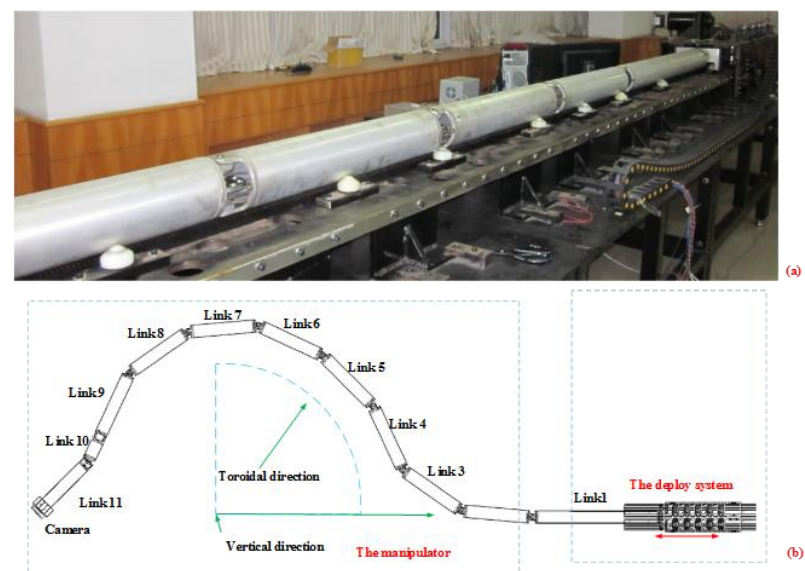

Fig. 2: The model of the long reach robot: (a) The prototype, (b)The 3D model

The long reach robot is mainly composed of the deploy system, the manipulator and the tool. It is $8 \mathrm{~m}$ long in full extension and has 11 DOFs, which is composed of the deploy system, the manipulator and the process tool.

The deploy system, which has $1 \mathrm{DOF}$, is contacted to the base via a prismatic joint. It is composed of the car, the rail, the gear and gear rack, which can support the robot and provide high precision displacement. The manipulator, which has 9 lateral DOFs and 1 vertical DOF, is contacted to the deploy system via a revolute joint. It is composed of 11 links and 10 joints, which can move along the designed trajectory and arrive at the desired location without collision. The inspection tool, which can be changed for the specific work, is installed on the end of the manipulator. The model of the long reach robot can be seen in Fig.2. 


\section{Static analysis}

The robot has the high cantilever structure while the tube diameter is limited, which means some components, especially the components near the base, are submitted high force and torque. It's essential to investigate the force performance of the device. The static analysis can be separated into two parts: the energy analysis, which is based on the virtual work principal, and the structural analysis, which is based on the single module static equilibrium.

\subsection{Energy analysis $[5,6]$}

The relation between the displacement and the work can be denoted as Eq.1

$$
\delta w=\sum_{i} f_{i} \delta x_{i} .
$$

According to the virtual work principle[5,6], if the virtual work done by applied force is zero in displacements consistent with constraints, it can be expressed as Eq.2

$$
\tau^{T} \delta q+(-F)^{T} \delta x=0
$$

Considering the instantaneous kinematics, it can be expressed as Eq.3

$$
\tau=J^{T} F
$$

Where $\tau$ is the torque acted at the joint, $\mathrm{F}$ is the generalized force applied over the end-effector, $\mathrm{J}^{\mathrm{T}}$ is the transpose of the Jacobian matrix [3,4]. Fig.3 shows the generalized force and torque applied over the end-effector and the joints, respectively.

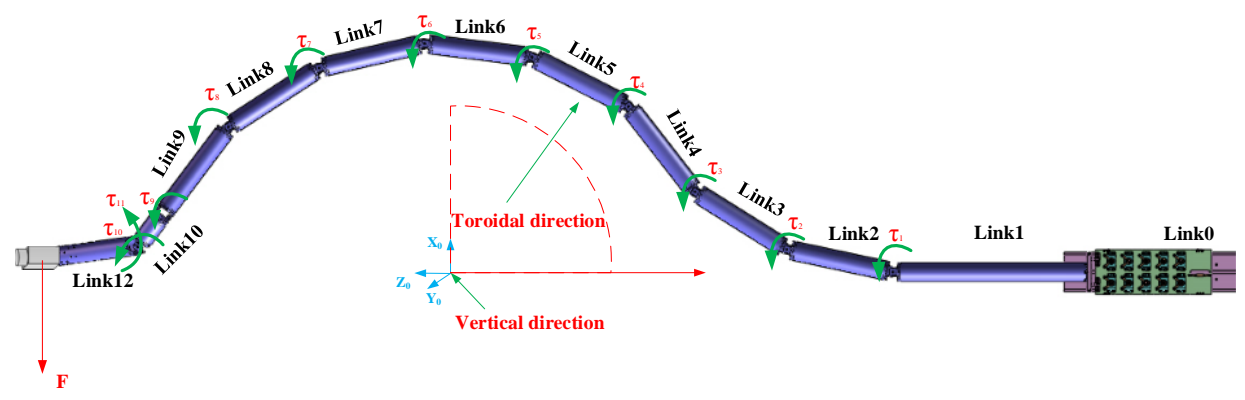

Fig. 3: Force and torque in the manipulator

\subsection{Structural analysis}

It is important to investigate the internal force and torque in all joints and links for providing reference for components selection and ensuring the safety of the long reach robot. Therefore, the static equilibrium is given in this paper. The force and torque caused by self-gravity and external load over one module of the long reach robot can be seen in Eq.4. 


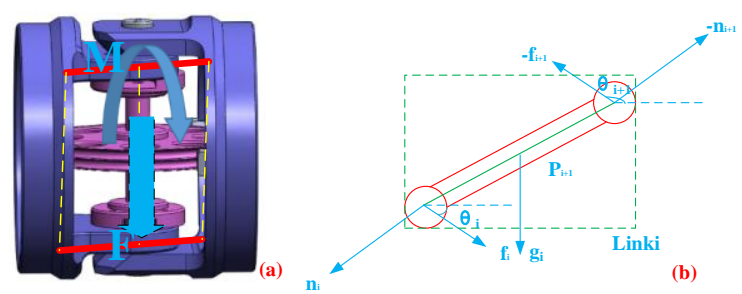

Fig. 4: Internal Force and torque: (a) The force in the joint, (b)The force on single module

Because of the symmetrical structure of the single module, the center of mass of the link is coincident with the centroid when the manufacturing and assembly errors is neglecting. Therefore, the gravity on the module can be seen a concentrated force act at the centroid.

According to static equilibrium about the centroid of joint $i$, the force and torque applied on the module can be expressed as Eq.4.

$$
\left\{\begin{array}{l}
\sum F_{i}=0, \sum M_{i}=0 \\
f_{i}=\frac{f_{i+1} \sin \theta_{i+1}}{\cos \theta_{i}}, n_{i}=n_{i+1}+P_{i+1} \times f_{i+1}-\frac{P_{i+1} \times g_{i}}{2}
\end{array} .\right.
$$

Where, $f_{i}$ and $n_{i}$ is the force and torque applied on the joint $i, g_{i}$ is the gravity of module $i, \theta_{i}$ and $\theta_{i+1}$ is the angle with respect to the horizontal line, $P_{i+1}$ is the vector from the centroid of joint $i$ to the centroid of joint $\mathrm{i}+1$.

The force and torque with respect to the frame attached to the link i can be expressed as Eq.5

$$
{ }^{i} f_{i}={ }_{i+1}^{i} R \frac{{ }^{i+1} f_{i+1} \sin \theta_{i+1}}{\cos \theta_{i}},{ }^{i} n_{i}={ }^{i}{ }_{i+1} R^{i+1} n_{i+1}+{ }^{i} P_{i+1} \times{ }^{i} f_{i+1}-\frac{{ }^{i} P_{i+1} \times g_{i}}{2}
$$

The force and torque applied on the end-effector is decided by the selection of the inspection tool and can be gotten. Besides, the gravity of the long reach robot decided by the design can be known. Thus, the force and torque applied over the joints and links can be gotten from the propagation based on the single module structural force analysis.

\subsection{Force Simulation for key components}

The long reach robot has the cantilever structure while the length in full extension is up to $8 \mathrm{~m}$, which means some components bear high force and torque. Considering the long reach robot is supported by the deploy system, the car and the guide rail are suffered higher force and torque than other components. Besides, the module mounted on the deploy system is suffered higher force and torque than other modules. It's essential to investigate the performance of the components mentioned above. In order to ensure the safety of the system, the simulation for key components, 
including the module near the base, the car and the guide rail, have been finished by the finite element(FE) method. The detailed simulation results can be seen in Fig.5. The stresses on the key components are much less than the allowed value of the selection material.

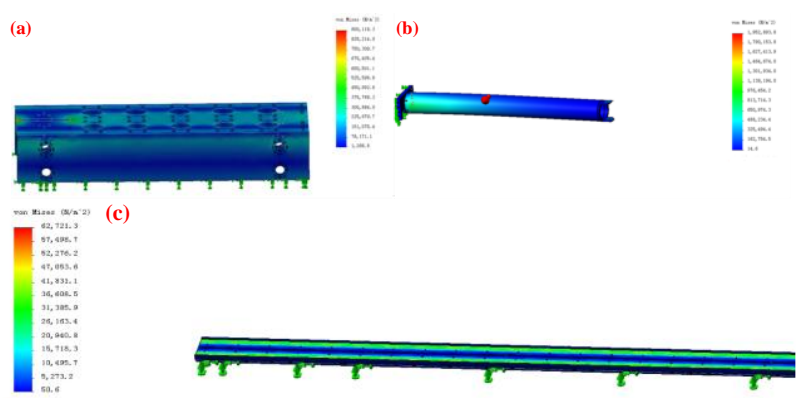

Fig. 5: Detailed force simulation: (a) car, (b) module near the base, (c) linear guide

\section{Conclusion}

The remote inspection robot has been designed and built. For studying the performance of the device during the inspection procedure, the force analysis is presented. Besides, the simulation for key components is given. The analysis and simulation mentioned above show that the robot has the ability to finish inspection task. In the next step, the upgraded prototype with more DOFs and small space occupied will be manufactured.

\section{Acknowledgments}

The works presented in this paper are funded by the National Science and Technology Projects (No. 2012GB102, 007) and the National Nature Science Foundation of China under Contract No. 61503361.The authors would like to thank the support and assistance from all people involved in the design, implementation and development of the long reach robot.

\section{Reference}

[1]S. Wu, An overview of the EAST project, Fusion Eng Des. 82(2007)463-471 .

[2]X. B. Peng, Y. T. Song, C. C. Li, M. Z. Lei, G. Li, Conceptual design of EAST flexible in-vessel inspection system, Fusion Eng Des. 85(2010)1362-1365.

[3]Z. Wu, B. Wu, Q. Zhang, Z. Wang, Development and Analysis of a Long Reach Robot for EAST Vacuum Vessel Inspection, J Fusion Energ. 34(2015)983-988.

[4]Z. Wu, B. Wu, Z. Wang, Kinematic and Dynamic Analysis of a In-Vessel Inspection Robot System for EAST, J Fusion Energ. 34(2015)1203-1209.

[5]B. Siciliano, O. Khatib, Springer handbook of robotics, first ed., Springer Science \& Business Media, New York,2008.

[6]Z. X. Cai, Robotics, second ed., Tsinghua University Press, Beijing, 2009. 